\title{
Endovascular Extraction of a Needle from the Internal Carotid Artery: A Novel Approach to a Controversial Dental Misadventure
}

\author{
Jonathan P. Giurintano ${ }^{1}$ Jessica Somerville ${ }^{1}$ Merry Sebelik $^{2}$ Daniel Hoit ${ }^{3} \quad$ L. Madison Michael III ${ }^{3}$ \\ Courtney B. Shires ${ }^{1}$
}

${ }^{1}$ Department of Otolaryngology-Head \& Neck Surgery, University of Tennessee Health Science Center, Memphis, Tennessee, United States

2 Department of Otolaryngology-Head \& Neck Surgery, Emory University, Atlanta, Georgia, United States

${ }^{3}$ Department of Neurosurgery, University of Tennessee Health Science Center, Memphis, Tennessee, United States

Address for correspondence Jonathan P. Giurintano, MD, Department of Otolaryngology-Head \& Neck Surgery University of Tennessee Health Science Center, 910 Madison Avenue, Suite 430, Memphis, TN 38163, United States

(e-mail: Jonathan.Giurintano@ucsf.edu).

J Neurol Surg Rep 2017;78:e106-e108.

\begin{abstract}
Keywords

- skull base

- endovascular

- dental needle

- internal carotid artery

Objective To review the literature concerning the management of dental needles broken off into the deep spaces of the neck, to report what we believe is the first case of a fractured dental needle migrating into the jugular foramen, and the unconventional use of endovascular intervention to retrieve the needle fragment.

Design Case report with review of literature.

Setting Academic tertiary care center.

Participants Intervention was performed by the otolaryngology-head and neck surgery, vascular surgery, and neurovascular interventional radiology teams.

Results Transoral exploration, including palatal split and exposure of the poststyloid parapharyngeal space with C-arm image guidance, was unable to retrieve the broken needle, which traversed the internal carotid lumen with the distal end entering the jugular foramen. Through endovascular intervention, the neurovascular interventional radiology team captured the proximal end of the needle and retrieved it through the femoral artery. The patient recovered uneventfully.

Conclusion Fracture and loss of oral injection needles remain a persistent and preventable problem. This case demonstrates a novel, minimally invasive, welltolerated, and successful method to extract a fractured needle that migrated into the lumen of the internal carotid artery at the level of the skull base.
\end{abstract}

\section{Introduction}

Regional block of the inferior alveolar nerve through intraoral injection of an anesthetic agent is a regular practice in the fields of dentistry and oral surgery in preparation for dental procedures and extractions, as it provides regional anesthesia to the structures of the mandibular (V3) nerve distribution, thereby facilitating intervention of the mandibular dentition.
Local anesthetic is traditionally administered through 27 - or 30 -gauge needles, penetrating the oral mucosa lateral to the mandibular ramus to enter the pterygomandibular space. Though regularly performed on thousands of patients each day without incident, a potential complication of this procedure is the accidental breakage and dislodgement of the needle into the submucosal soft tissue of the pterygomandibular space. This complication is well documented in the dental received

November 23, 2016 accepted after revision May 8, 2017
DOI https://doi.org/

10.1055/s-0037-1604282. ISSN 2193-6358. (c) 2017 Georg Thieme Verlag KG
Stuttgart · New York

License terms

(c) $(1) \$$ 


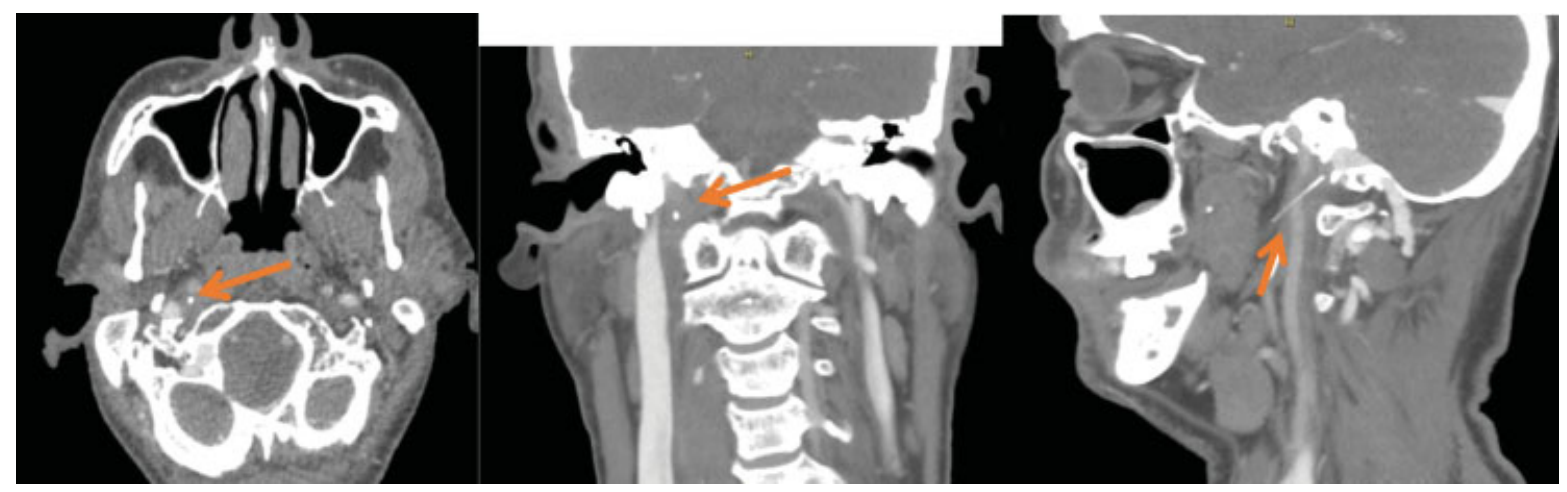

Fig. 1 Preoperative computed tomography (CT) imaging.

and medical literature, with Blum reporting a review of 165 cases as early as $1924^{1,2}$ As the standards for manufacturing hypodermic needles has improved, the incidence of fractured dental needles has nearly been eliminated; however, case reports detailing this complication continue to appear in the literature. Reported risk factors for needle fracture include the use of 30-gauge needles, bending the needle prior to injection, "hubbing" the needle against the mucosa during injection, administering multiple injections with the same needle, and sudden patient movement during injection. ${ }^{3,4}$ Over the course of history, management recommendations have varied from observation only to immediate removal in the operating room through transoral or transcervical surgical approaches. ${ }^{5}$ As it has been observed that over time, the action of the masticatory muscles can cause the retained needles to migrate through the pterygomandibular space into the deep spaces of the neck and even as far as the cochlea, ${ }^{6}$ the recent literature recommends immediate removal under general anesthesia. We present the case of a patient who presented to our institution with a retained dental needle that had migrated into the carotid sheath at the level of the skull base, and the unique approach that succeeded in the retrieval of the needle.

\section{Case Report}

A 57-year-old male patient from a small community underwent multiple dental extractions by his local general dentist.
One day after the procedure, he was notified that a needle was missing from the count and could possibly be retained in the oral soft tissues. As he was asymptomatic at the time, the dentist instructed him that no acute intervention was necessary. Over the course of 4 weeks, he developed progressively worsening right neck pain, dysphagia, and trismus, prompting him to seek care at an emergency department. Computed tomography (CT) imaging revealed a linear metallic object penetrating the lumen of the internal carotid artery (ICA) near the skull base ( $\mathbf{- F i g . 1}$ ). The patient was then transferred to our tertiary referral center. On initial physical examination, the patient was found to have less than $1 \mathrm{~cm}$ trismus with severe pain over the right side of his neck. A multidisciplinary surgical team (vascular surgery, otolaryngology-head and neck surgery, neurosurgery, and oral surgery) was assembled, and the following day, the otolaryngology-head and neck surgery team, with the assistance of vascular surgery team, performed transoral exploration, including palatal split and lateral pharyngotomy, to explore the poststyloid parapharyngeal space with C-arm image guidance. The foreign body was unable to be visualized or palpated, and as such, a tracheostomy was performed to secure the airway and the attempted removal was aborted. Postoperative imaging revealed that the needle had further migrated and was now traversing the internal carotid lumen with the distal end entering the jugular foramen (-Fig. 2). As there was a concern for thrombus encompassing the needle fragment within the ICA and risk of cerebrovascular

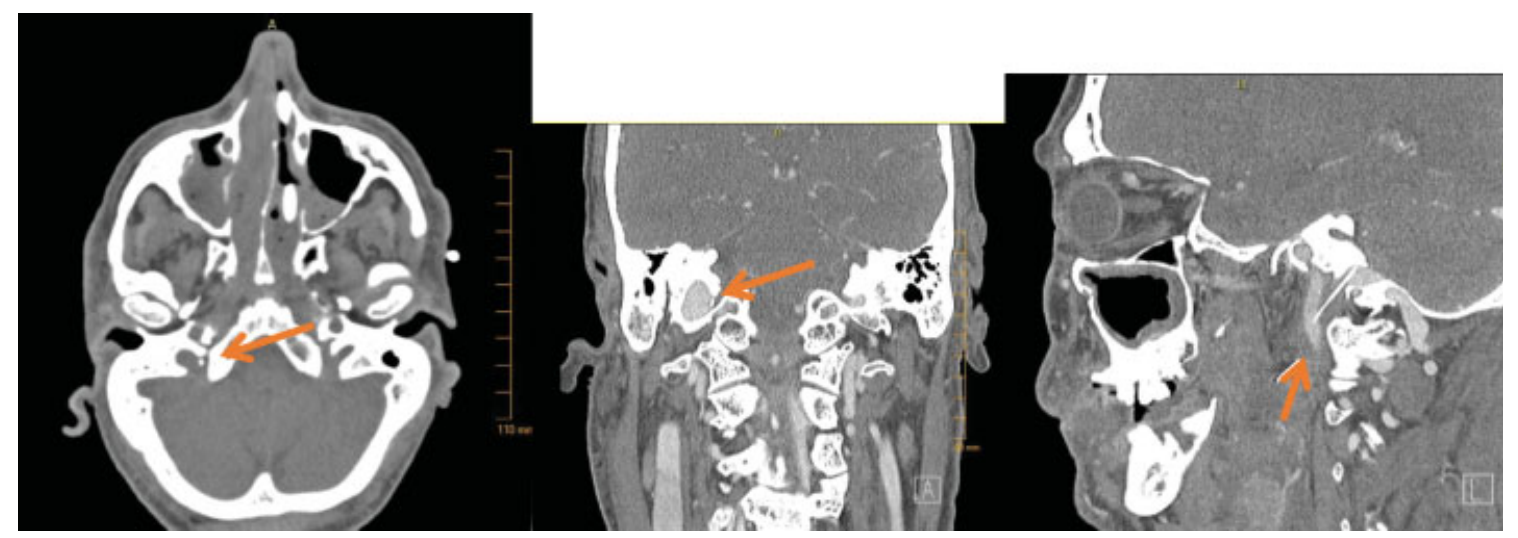

Fig. 2 Postoperative computed tomography (CT) imaging. 
accident, prior to continuing with a more invasive open surgical approach to access the skull base, the neurovascular interventional radiology team was requested to perform a cerebral angiogram.

Two days after the failed transoral attempt at needle removal, the neurovascular interventional radiology team at our institution performed a cerebral angiogram via femoral arterial approach to evaluate the position of the foreign body within the carotid artery. During the angiogram, the team identified a foreign body that appeared to be a needle located in the right ICA at approximately the level of the first cervical vertebra. The team recognized that the proximal end of the needle was protruding from the posterior wall of the ICA. Using a 4-mm GooseNeck Microsnare, the interventional radiologists grasped the needle within the ICA, slowly removing it from the ICA into a balloon catheter. Once removed, the needle was identified within the catheter and sent to pathology. Postretrieval angiogram demonstrated a right grade I carotid dissection with no flow impact, and as such, no further intervention was performed. The patient recovered from the procedure uneventfully with no cerebrovascular complications; his tracheostomy tube was decannulated in the immediate postoperative period; and he was subsequently discharged home on anticoagulation. He had no further complications.

\section{Discussion}

Despite the safety of modern stainless steel hypodermic needles, the fracture and loss of needles in the pterygomandibular space during inferior alveolar nerve block remain a complication. Though the literature suggests that needle breakage is largely prevented by the use of 27-gauge needles and proper technique (no prebending of the needle, no hubbing of the needle, etc), its occurrence is not entirely preventable, and while immediate retrieval under general anesthesia is the current treatment recommendation, delayed intervention may allow migration of the needle into the deep spaces of the neck, precluding extraction through a transoral approach. In the senior authors' (M.S., C.S.) experience, needle retrieval through a transoral approach is difficult and often unsuccessful, particularly if the retrieval attempt is not performed immediately after loss of the needle. While a transcervical approach with mandibulotomy offers wide exposure of the carotid vasculature at its entry into the skull base, this approach exposes the patient to significant surgical morbidity and risks, including infection, salivary fistula, malocclusion, and nonunion.
In the case of this patient, it was recognized that the needle was not completely traversing the lumen of the ICA; rather, a segment of the needle remained free within the lumen of the artery. This characteristic provided a free end that allowed the needle to be successfully grasped and removed through the femoral artery using the endovascular approach, saving this patient the potential morbidity of a much more invasive open procedure (mandibulotomy) to access the skull base.

\section{Conclusion}

With the advent of advanced imaging and the ability of lost dental needles to drastically migrate to difficult to access locations over the course of only few weeks, it is recommended that lost dental needles be retrieved immediately by an experienced surgical team under general anesthesia, as a delayed transoral approach is difficult and often unsuccessful. This case demonstrates a novel, minimally invasive, welltolerated, and successful method that was performed to retrieve a fractured dental needle that had migrated into the lumen of the ICA and penetrated the jugular foramen. If the retained needle is located within the lumen of the ICA, as it was in our case, then an endovascular approach to retrieve it is feasible and has been shown to be successful.

\section{References}

1 Blum T. Further observations with hypodermic needles broken during the administration of oral local anesthesia: a report of 65 cases. Dent Cosmos 1924;66:322-328

2 Blum T. A report of 100 cases of hypodermic needles broken during administration of oral local anesthesia. Dent Cosmos 1928;70:865-874

3 Pogrel MA. Broken local anesthetic needles: a case series of 16 patients, with recommendations. J Am Dent Assoc 2009; 140(12):1517-1522

4 Augello M, von Jackowski J, Grätz KW, Jacobsen C. Needle breakage during local anesthesia in the oral cavity-a retrospective of the last 50 years with guidelines for treatment and prevention. Clin Oral Investig 2011;15(01):3-8

5 Zeltser R, Cohen C, Casap N. The implications of a broken needle in the pterygomandibular space: clinical guidelines for prevention and retrieval. Pediatr Dent 2002;24(02):153-156

6 Casey JT, Lupo JE, Jenkins HA. Retained dental needle migration across the skull base to the cochlea presenting as hearing loss. Otol Neurotol 2015;36(02):e42-e45 